\title{
A General Multi-agent Epistemic Planner Based on Higher-order Belief Change
}

\author{
Xiao Huang, Biqing Fang, Hai Wan, Yongmei Liu \\ Dept. of Computer Science, Sun Yat-sen University, Guangzhou 510006, China \\ \{huangx233,fangbq3\}@mail2.sysu.edu.cn, \{wanhai,ymliu\}@mail.sysu.edu.cn
}

\begin{abstract}
In recent years, multi-agent epistemic planning has received attention from both dynamic logic and planning communities. Existing implementations of multi-agent epistemic planning are based on compilation into classical planning and suffer from various limitations, such as generating only linear plans, restriction to public actions, and incapability to handle disjunctive beliefs. In this paper, we propose a general representation language for multi-agent epistemic planning where the initial $\mathrm{KB}$ and the goal, the preconditions and effects of actions can be arbitrary multi-agent epistemic formulas, and the solution is an action tree branching on sensing results. To support efficient reasoning in the multi-agent KD45 logic, we make use of a normal form called alternating cover disjunctive formulas (ACDFs). We propose basic revision and update algorithms for ACDFs. We also handle static propositional common knowledge, which we call constraints. Based on our reasoning, revision and update algorithms, adapting the PrAO algorithm for contingent planning from the literature, we implemented a multi-agent epistemic planner called MEPK. Our experimental results show the viability of our approach.
\end{abstract}

\section{Introduction}

Many intelligent tasks involve the interaction of multiple agents, raising the need of reasoning about knowledge and beliefs, and their change. For illustration, consider the following example from [Kominis and Geffner, 2015]. There is a corridor of several rooms, and a number of boxes that are in some of the rooms. Two agents can move along this corridor. The task is for the two agents to collaborate via communication in finding out the positions of the boxes. Performance of actions results in the change of not only the world but also agents' knowledge and beliefs. In some cases, it is even necessary to reason about higher-order knowledge and beliefs, i.e., knowledge and beliefs about other agents' knowledge and beliefs. For example, agent $a$ may wish to know a secret $s$ with agent $b$ knowing she knows $s$, or without $b$ knowing she knows $s$, or even with $b$ believing she does not know $s$.
In recent years, multi-agent epistemic planning has received attention from both dynamic logic and planning communities. On the theory side, Bolander and Andersen [2011] formalized multi-agent epistemic planning based on dynamic epistemic logic (DEL) [van Ditmarsch et al., 2007] and showed that it is undecidable in general. In the DEL-based formalization, states are represented as Kripke models, and actions are represented as action models, which are Kripke models of actions, describing agents' abilities to distinguish among actions. Further, Aucher and Bolander [2013] showed that multi-agent epistemic planning is undecidable in the presence of only purely epistemic actions. Meanwhile, Yu et al. [2013] identified two important decidable fragments of multi-agent epistemic planning. On the implementation side, Kominis and Geffner [2015], and Muise et al. [2015] showed how to exploit classical planning to solve restricted versions of multi-agent epistemic planning problems. By resorting to classical planning, both methods can only generate linear plans, doing conformant planning. Moreover, Kominis and Geffner assumed all actions are public, and hence dealt with knowledge. In contrast, Muise et al. focused on beliefs; however, they can only handle bounded-depth belief literals, disallowing disjunctive beliefs. In the single-agent case, Wan et al. [2015] presented a complete epistemic planner without the epistemic closed world assumption. They proposed normal forms for epistemic formulas which support tractable reasoning and progression, and adapted the PrAO algorithm for contingent planning [To et al., 2011].

Belief change studies how an agent modifies her beliefs on receiving new information. Two main types of belief change are revision and update: revision concerns belief change about static environments due to partial and possibly incorrect information, whereas update concerns belief change about dynamic environments due to the performance of actions. Various guidelines for belief change have been proposed, and the most popular ones are the AGM postulates for belief revision [Alchourrón et al., 1985], the KM postulates for belief update [Katsuno and Mendelzon, 1991], and the DP postulates for iterated belief revision [Darwiche and Pearl, 1997]. Katsuno and Mendelzon [1991] briefly discussed how belief revision and update can be used for reasoning about actions: if a condition $\phi$ is found true, the KB is revised with $\phi$; if an action with postcondition $\psi$ is performed, the $\mathrm{KB}$ is updated with $\psi$. There has been work extending belief change 
from propositional logic to epistemic logic. Aucher [2010] generalized the AGM postulates to multi-agent settings. Also, Aucher [2011] explored the progression of KBs wrt actions, where both KBs and actions are represented as canonical formulas in modal logics, which capture Kripke models up to certain depths [Moss, 2007]. Recently, Caridroit et al. [2016] investigated several measures of distances between $\mathrm{KD} 45_{n}$ Kripke models, and use them to define the revision of beliefs represented by such models. Finally, Miller and Muise [2016] studied belief update for KBs consisting of belief literals.

In this paper, we consider multi-agent epistemic planning from the viewpoint of a third person. We treat contingent rather than conformant planning, resulting in nonlinear plans. We model private actions and hence handle beliefs, formalized with the multi-agent modal logic $\mathrm{KD} 45_{n}$ [Fagin et al., 1995]. We do not support arbitrary common knowledge, but we handle static propositional common knowledge, which we call constraints. For such planning settings, we propose a general representation framework where the initial KB and the goal, the preconditions and effects of actions can be arbitrary $\mathrm{KD} 45_{n}$ formulas, progression of KBs wrt actions is achieved through the operation of belief revision or update, and the solution is an action tree branching on sensing results. To support efficient reasoning and progression, we make use of a normal form for $\mathrm{KD} 45_{n}$ called alternating cover disjunctive formulas (ACDFs) [Hales et al., 2012]. We propose basic revision and update algorithms for ACDF formulas. Based on our reasoning, revision and update algorithms, adapting the PrAO algorithm, we implemented a multi-agent epistemic planner called MEPK. Our experimental results show the viability of our approach.

\section{Preliminaries}

In this section, we introduce the background work of our paper, i.e., the multi-agent modal logic $\mathrm{KD} 45_{n}$, alternating cover disjunctive formulas, and belief revision and update.

\subsection{Multi-agent Modal Logic KD45}

Consider a finite set of agents $\mathcal{A}$ and a finite set of atoms $\mathcal{P}$. We use $\phi$ and $\psi$ for formulas, $\Phi$ and $\Psi$ for sets of formulas.

Definition 2.1 The language $\mathcal{L}_{K C}$ of multi-agent modal logic with common knowledge is generated by the BNF:

$$
\varphi::=p|\neg \phi|(\phi \wedge \psi)\left|K_{a} \phi\right| C \phi, \text { where }
$$

$p \in \mathcal{P}, a \in \mathcal{A}, \phi, \psi \in \mathcal{L}_{K C}$. We use $\mathcal{L}_{K}$ for the language without the $C$ operator, and $\mathcal{L}_{0}$ for the propositional language.

Intuitively, $C \phi$ means commonly knowing $\phi$. In this paper, we restrict our attention to the case of propositional common knowledge, i.e., $C \phi$ where $\phi \in \mathcal{L}_{0}$, and we call $\phi$ a constraint. We use $C^{*} \phi$ to denote $\phi \wedge C \phi$.

We let $L_{a} \phi$ stand for $\neg K_{a} \neg \phi$. We let $\top$ and $\perp$ represent true and false respectively. We let $\bigvee \Phi$ (resp. $\bigwedge \Phi$ ) denote the disjunction (resp. conjunction) of members of $\Phi$; and we use $L_{a} \Phi$ to represent the conjunction of $L_{a} \phi$ where $\phi \in \Phi$. The modal depth of a formula $\phi$ in $\mathcal{L}_{K}$ is the depth of nesting of modal operators in $\phi$.
Definition 2.2 A frame is a pair $(W, R)$, where $W$ is a nonempty set of possible worlds; for each agent $a \in \mathcal{A}, R_{a}$ is a binary relation on $W$, called the accessibility relation for $a$.

We say $R_{a}$ is serial if for any $w \in W$, there is $w^{\prime} \in W$ s.t. $w R_{a} w^{\prime}$; we say $R_{a}$ is Euclidean if whenever $w R_{a} w_{1}$ and $w R_{a} w_{2}$, we get $w_{1} R_{a} w_{2}$. A KD $45_{n}$ frame is a frame whose accessibility relations are serial, transitive and Euclidean.

Definition 2.3 A Kripke model is a triple $M=(W, R, V)$, where $(W, R)$ is a frame, and $V$ is a valuation map, which maps each $w \in W$ to a subset of $\mathcal{P}$. A pointed Kripke model is a pair $s=(M, w)$, where $M$ is a Kripke model and $w$ is a world of $M$, called the actual world.

Definition 2.4 Let $s=(M, w)$ be a Kripke model where $M=(W, R, V)$. We interpret formulas in $\mathcal{L}_{K C}$ by induction:

- $M, w \models p$ iff $p \in V(w)$;

- $M, w \models \neg \phi$ iff $M, w \not \models \phi$;

- $M, w \models \phi \wedge \psi$ iff $M, w \models \phi$ and $M, w \models \psi$;

- $M, w \models K_{a} \psi$ iff for all $v$ s.t. $w R_{a} v, M, v \models \phi$;

- $M, w \models C \phi$ iff for all $v$ s.t. $w R_{\mathcal{A}} v, M, v \models \phi$, where $R_{\mathcal{A}}$ is the transitive closure of the union of $R_{a}$ for $a \in \mathcal{A}$.

We say $\phi$ is satisfiable if there is a $\operatorname{KD} 45_{n} \operatorname{model}(M, w)$ s.t. $M, w \models \phi$. We say $\phi$ entails $\psi$, written $\phi \models \psi$, if for any model $(M, w), M, w \models \phi$ implies $M, w \models \psi$. We say $\phi$ and $\psi$ are equivalent, written $\phi \Leftrightarrow \psi$, if $\phi \models \psi$ and $\psi \models \phi$.

We say that $\phi$ is satisfiable wrt constraint $\gamma \in \mathcal{L}_{0}$ if $\phi \wedge C^{*} \gamma$ is satisfiable; we say that $\phi$ entails $\psi$ wrt constraint $\gamma$, written $\phi=_{\gamma} \psi$, if $\phi \wedge C^{*} \gamma \models \psi \wedge C^{*} \gamma$.

\subsection{Alternating Cover Disjunctive Formulas}

Janin and Walukiewicz [1995] introduced the notion of disjunctive formulas for modal $\mu$-calculus and showed that every formula is equivalent to a disjunctive formula. D'Agostino and Lenzi [2006] gave the definition of disjunctive formulas for modal logics, using a cover modality. Ten Cate et al. [2006] showed that every formula in the multi-agent modal $\operatorname{logic} \mathrm{K}_{n}$ is equivalent to a disjunctive formula whose length is at most singly exponential in the length of the original formula. We slightly vary the definition, and use the name cover disjunctive formulas.

Definition 2.5 Let $a \in \mathcal{A}$, and $\Phi$ a set of formulas. We use $\nabla_{a} \Phi$ to denote the formula $K_{a}(\bigvee \Phi) \wedge L_{a} \Phi$.

We use $\Phi \wedge \psi$ to denote the set $\{\phi \wedge \psi \mid \phi \in \Phi\}$.

Proposition 2.6 1. $\nabla_{a}\{\top\} \Leftrightarrow \top$;

2. $K_{a} \psi \wedge L_{a} \Phi \Leftrightarrow \nabla_{a}(\{\psi\} \cup(\Phi \wedge \psi))$;

3. $\nabla_{a} \Phi \wedge \nabla_{a} \Phi^{\prime} \Leftrightarrow \nabla_{a}\left[\Phi \wedge\left(\bigvee \Phi^{\prime}\right) \cup \Phi^{\prime} \wedge(\bigvee \Phi)\right]$.

Definition 2.7 The set of cover disjunctive formulas (CDFs) is inductively defined as follows:

1. A propositional term, i.e., a conjunction of propositional literals, is a CDF;

2. If $\phi_{0}$ is a propositional CDF, and for each $a \in \mathcal{B} \subseteq \mathcal{A}$, $\Phi_{a}$ is a finite set of CDFs, then $\phi_{0} \wedge \bigwedge_{a \in \mathcal{B}} \nabla_{a} \Phi_{a}$ is a $\mathrm{CDF}$, called a CDF term; 
3. If $\Phi$ is a non-empty finite set of CDF terms, then $\bigvee \Phi$ is a CDF, called a disjunctive $\mathrm{CDF}$.

So a propositional CDF is in DNF (disjunctive normal form).

Moss [2007] introduced the concept of canonical formulas, very similar to formulas introduced by Fine [1975], and showed that every formula in $\mathrm{K}_{n}$ is equivalent to a disjunction of a finite set of canonical formulas. However, in the definition of canonical formulas, no disjunction is allowed. As a result, the conversion may cause a non-elementary blowup in size, as compared to a single exponential blowup for CDFs.

We now define a complexity measure $\|\cdot\|$ for CDFs.

Definition 2.8 The complexity measure $\|\cdot\|$ for CDFs is inductively defined as follows:

1. $\|t\|=0$, where $t$ is a propositional term;

2. $\left\|\phi_{0} \wedge \bigwedge_{a \in \mathcal{B}} \nabla_{a} \Phi_{a}\right\|=\Sigma_{a \in \mathcal{B}}\left(1+\Sigma_{\phi \in \Phi_{a}}\|\phi\|\right)$;

3. $\|\bigvee \Phi\|=\Sigma_{\phi \in \Phi}\|\phi\|$.

Hales et al. [2012] introduced the notion of alternating cover disjunctive formulas, and showed that in $\mathrm{KD} 45_{n}$, every formula in $\mathcal{L}_{K}$ is equivalent to such a formula.

Definition 2.9 We say that a formula has the alternating agent modality property if it has the property that modal operators of an agent do not directly occur inside those of the same agent. We call a CDF with the alternating agent modality property an alternating cover disjunctive formula (ACDF).

For example, $\nabla_{a}\{\top, q\} \wedge \nabla_{b}\left\{\top, \nabla_{a}\{\top, \neg q\}\right\}$ is an ACDF, but the $\operatorname{CDF} \nabla_{a}\left\{\neg p \wedge q, \nabla_{b}\{p, q\}, \nabla_{a}\{\neg p \wedge q\}\right\}$ is not.

Proposition 2.10 In KD45n, every formula in $\mathcal{L}_{K}$ can be transformed to an equivalent $A C D F$ whose length is at most singly exponential in the length of the original formula.

Proof: We first convert the formula into negation normal for$\mathrm{m}$, and then we apply the following transformation rules until the formula satisfies the alternating agent modality property: $K_{a}(\phi \wedge \psi) \Leftrightarrow K_{a} \phi \wedge K_{a} \psi, L_{a}(\phi \vee \psi) \Leftrightarrow L_{a} \phi \vee L_{a} \psi ;$ $L_{a}\left(\phi \wedge K_{a} \psi\right) \Leftrightarrow L_{a} \phi \wedge K_{a} \psi, L_{a}\left(\phi \wedge L_{a} \psi\right) \Leftrightarrow L_{a} \phi \wedge L_{a} \psi ;$ $K_{a}\left(\phi \vee K_{a} \psi\right) \Leftrightarrow K_{a} \phi \vee K_{a} \psi, K_{a}\left(\phi \vee L_{a} \psi\right) \Leftrightarrow K_{a} \phi \vee L_{a} \psi$. Now by Lemma 7 of [ten Cate et al., 2006], this formula can be converted to an equivalent CDF with at most a single exponential blowup in size. Since the conversion preserves the alternating agent modality property, the result is an ACDF.

A modal term is a conjunction of propositional formulas and modal atoms of the form $K_{a} \phi$ or $L_{a} \phi$. We call a modal term with the alternating agent modality property an alternating modal term. In the following, we present a result concerning how to check satisfiability of alternating modal terms and its easy corollaries, which will be used later in our paper.

Proposition 2.11 An alternating modal term $\delta=$ $\phi_{0} \wedge \bigwedge_{a \in \mathcal{B}}\left(K_{a} \phi_{a} \wedge L_{a} \Psi_{a}\right)$ is satisfiable wrt constraint $\gamma$ iff the following hold:

1. $\phi_{0} \wedge \gamma$ is propositionally satisfiable;

2. for each $a \in \mathcal{B}, \phi_{a}$ is satisfiable wrt $\gamma$;

3. for each $a \in \mathcal{B}$, for each $\psi \in \Psi_{a}, \phi_{a} \wedge \psi$ is satisfiable wrt $\gamma$.
Proof: The only-if direction is easy; we prove the if direction. Since $L_{a} \top \Leftrightarrow \top$, without loss of generality, we assume that for each $a \in \mathcal{B}, \Psi_{a}$ is not empty. Construct a model $(M, w)$ as follows. By condition 1, create a new world $w$ satisfying $\phi_{0} \wedge \gamma$. By condition 3, for each $a \in \mathcal{B}$, for each $\psi \in \Psi_{a}$, there is a $\operatorname{KD} 45_{n}$ model $\left(M_{\psi}, w_{\psi}\right)$ satisfying $\phi_{a} \wedge \psi \wedge C^{*} \gamma$, add a new copy of $\left(M_{\psi}, w_{\psi}\right)$ into $M$, and let $w R_{a} w_{\psi}$; then add $a$-edges between all the $a$-children of $w$. Since $\delta$ is an alternating modal term, $\phi_{a}$ and $\psi \in \Psi_{a}$ do not use $K_{a}$ or $L_{a}$ as outmost modalities. Also, all worlds of $M$ satisfy $\gamma$. Thus $M, w_{\psi} \models \phi_{a} \wedge \psi \wedge C^{*} \gamma$. So $(M, w)$ is a $\mathrm{KD}_{4} 5_{n}$ model satisfying $\delta$.

As easy corollaries, we get

Proposition 2.12 Let $\delta=\phi_{0} \wedge \bigwedge_{a \in \mathcal{A}}\left(K_{a} \phi_{a} \wedge L_{a} \Psi_{a}\right)$ and $\delta^{\prime}=\phi_{0}^{\prime} \wedge \bigwedge_{a \in \mathcal{A}}\left(K_{a} \phi_{a}^{\prime} \wedge L_{a} \Psi_{a}^{\prime}\right)$ be two alternating modal terms satisfiable wrt constraint $\gamma$. Then $\delta \models_{\gamma} \delta^{\prime}$ iff the following hold:

1. $\phi_{0} \wedge \gamma \models \phi_{0}^{\prime}$ propositionally;

2. for each $a \in \mathcal{A}, \phi_{a} \models_{\gamma} \phi_{a}^{\prime}$;

3. for each $a \in \mathcal{A}$, for every $\psi^{\prime} \in \Psi_{a}^{\prime}$ there is a $\psi \in \Psi_{a}$ s.t. $\phi_{a} \wedge \psi \models_{\gamma} \psi^{\prime}$.

Proposition 2.13 An ACDF term $\phi_{0} \wedge \bigwedge_{a \in \mathcal{B}} \nabla_{a} \Phi_{a}$ is satisfiable wrt a DNF $\gamma$ iff the following hold:

1. $\phi_{0} \wedge \gamma$ is propositionally satisfiable;

2. for all $a \in \mathcal{B}, \Phi_{a}$ is not empty;

3. for all $a \in \mathcal{B}$ and all $\phi \in \Phi_{a}$, $\phi$ is satisfiable wrt $\gamma$.

Thus it is tractable to check the satisfiability of ACDFs.

\subsection{Belief Revision and Update}

We use $\circ$ to denote a revision operator, and $\diamond$ an update operator. Let $\psi$ be the original formula, and $\mu$ the revision or update formula. To formalize the distinction between revision and update, Katsuno and Mendelzon [1991] presented model-theoretic definitions of them: intuitively, $\psi \circ \mu$ selects from the models of $\mu$ those that are closest to models of $\psi$, while $\psi \diamond \mu$ selects, for each model $M$ of $\psi$, the set of models of $\mu$ that are closest to $M$. As easy properties of the definitions: when $\psi \wedge \mu$ is satisfiable, $\psi \circ \mu$ is equivalent to $\psi \wedge \mu$; update is distributive over the initial formula, i.e., $\left(\psi_{1} \vee \psi_{2}\right) \diamond \mu$ is equivalent to $\left(\psi_{1} \diamond \mu \vee \psi_{2} \diamond \mu\right)$.

Let's illustrate the difference between revision and update with an example. Take the notion of closeness based on set inclusion, that is, a model $I$ is closer to $M$ than a model $J$ if $\operatorname{Diff}(I, M) \subseteq \operatorname{Diff}(J, M)$, where $\operatorname{Diff}(I, M)$ is the set of atoms where $I$ and $M$ assign different truth values. Then the above model-theoretic definitions give us Satoh's revison operator $\circ_{s}\left[\right.$ Satoh, 1988] and Winslett's update operator $\diamond_{w}$ [Winslett, 1988]. For example, let $\psi=(a \wedge b \wedge c) \vee(a \wedge$ $\neg b \wedge \neg c$ ), and $\mu=a \wedge c$. The models of $\psi$ are $M_{1}=\{a, b, c\}$ and $M_{2}=\{a, \neg b, \neg c\}$; the models of $\mu$ are $M_{1}$ and $M_{3}=$ $\{a, \neg b, c\}$. Then $\psi \circ_{s} \mu=a \wedge b \wedge c$, equivalent to $\psi \wedge \mu$, since $M_{1}$ is closet to models of $\psi$. On the other hand, $\psi \diamond_{w} \mu=$ $a \wedge c$, since $M_{1}$ is closest to $M_{1}$, but $M_{3}$ is closest to $M_{2}$. 


\section{Our Modeling Framework}

In this section, we present our modeling framework for multiagent epistemic planning (MEP), which is adapted from that for single-agent epistemic planning in [Wan et al., 2015].

We illustrate our framework with the collaboration via communication example from the introduction.

Example 1 There is a corridor of three rooms $p_{1}, p_{2}$ and $p_{3}$. Two boxes $b_{1}$ and $b_{2}$ are located in some of the rooms. Two agents 1 and 2 can move back and forth along this corridor. When an agent gets into a room, he can see if a box is in the room. An agent can communicate information to another agent. Initially, the two agents are in $p_{2}$ and the two boxes are not there. The goal is for agent 1 to know the position of $b_{1}$, and for agent 2 to know the position of $b_{2}$.

We begin with the definition of MEP problems, then give the definition of different kinds of actions and the associated progression operations, and end with the definition of MEP solutions. The actions we consider include ontic, communication and sensing actions. The first two kinds share the same representation, and we call them deterministic actions.

Definition 3.1 A multi-agent epistemic planning problem $\mathcal{Q}$ is a tuple $\langle\mathcal{A}, \mathcal{P}, \mathcal{D}, \mathcal{S}, \mathcal{I}, \mathcal{G}, \gamma\rangle$, where $\mathcal{A}$ is a set of agents; $\mathcal{P}$ is a set of atoms; $\mathcal{D}$ is a set of deterministic actions; $\mathcal{S}$ is a set of sensing actions; $\mathcal{I} \in \mathcal{L}_{\mathcal{K}}$ is the initial $\mathrm{KB} ; \mathcal{G} \in \mathcal{L}_{\mathcal{K}}$ is the goal; and $\gamma \in \mathcal{L}_{0}$ is the constraint.

The main reason we consider constraints in this paper is for natural modeling of planning domains: we use constraints to express static propositional common knowledge, as will be illustrated in our formalization of Example 1.

We now formalize Example 1.

- The atoms are: at $(i, p)$, meaning agent $i$ is in room $p$; and $i n(b, p)$, meaning box $b$ is in room $p$.

- The ontic actions are: left( $i)$, agent $i$ moves left; and $\operatorname{right}(i), i$ moves right.

- The communication actions are: $\operatorname{tell}(i, j, b, p)$, agent $i$ tells $j$ whether $b$ is in $p$.

- The sensing actions are: $\operatorname{find}(i, b, p): i$ sees if $b$ is in $p$.

- The initial KB is at $\left(1, p_{2}\right) \wedge a t\left(2, p_{2}\right) \wedge \neg i n\left(b_{1}, p_{2}\right) \wedge$ $\neg i n\left(b_{2}, p_{2}\right) \wedge K_{1}\left(a t\left(1, p_{2}\right) \wedge \neg i n\left(b_{1}, p_{2}\right) \wedge \neg i n\left(b_{2}, p_{2}\right)\right) \wedge$ $K_{2}\left(a t\left(2, p_{2}\right) \wedge \neg i n\left(b_{1}, p_{2}\right) \wedge \neg i n\left(b_{2}, p_{2}\right)\right)$;

- The goal is $\bigwedge_{i=1}^{2}\left(K_{i} i n\left(b_{i}, p_{1}\right) \vee K_{i} i n\left(b_{i}, p_{2}\right) \vee\right.$ $\left.K_{i} i n\left(b_{i}, p_{3}\right)\right)$, meaning agent 1 knows the position of box $b_{1}$, and agent 2 knows the position of box $b_{2}$.

- The constraint is $\gamma_{1} \wedge \gamma_{2}$, where $\gamma_{1}=$ $\bigwedge_{i=1}^{2}\left(a t\left(i, p_{1}\right) \wedge \neg a t\left(i, p_{2}\right) \wedge \neg a t\left(i, p_{3}\right) \vee \neg a t\left(i, p_{1}\right) \wedge\right.$ $\left.\operatorname{at}\left(i, p_{2}\right) \wedge \neg a t\left(i, p_{3}\right) \vee \neg a t\left(i, p_{1}\right) \wedge \neg a t\left(i, p_{2}\right) \wedge a t\left(i, p_{3}\right)\right)$, meaning each agent is at exactly one room, and $\gamma_{2}$ is similar, representing each box is in exactly one room.

The reason that we have in the initial KB at $\left(1, p_{2}\right) \wedge$ $K_{1} a t\left(1, p_{2}\right)$ instead of simply $K_{1} a t\left(1, p_{2}\right)$ is that we model beliefs rather than knowledge.

Definition 3.2 A deterministic action is a pair $\langle$ pre, eff $\rangle$, where pre $\in \mathcal{L}_{K}$ is the precondition; eff is a set of conditional effects, each of which is a pair $\langle c o n, c e f\rangle$, where con, cef $\in \mathcal{L}_{K}$ indicate the condition and the effect, respectively.
For example, left $(i)=\left\langle\right.$ pre, $\left\{\right.$ ef $f_{1}$, ef $\left.\left.f_{2}\right\}\right\rangle$, where pre $=\neg a t\left(i, p_{1}\right) \wedge K_{i} \neg a t\left(i, p_{1}\right)$, ef $f_{1}=\left\langle a t\left(i, p_{2}\right)\right.$, at $\left.\left(i, p_{1}\right) \wedge K_{i} a t\left(i, p_{1}\right)\right\rangle$, and $e f f_{2}$ is similarly defined. For another example, $\operatorname{tell}(i, j, b, p)=\left\langle\right.$ pre, $\left\{\right.$ eff $f_{1}$, ef $\left.\left.f_{2}\right\}\right\rangle$, where pre $=K_{i} i n(b, p) \vee K_{i} \neg i n(b, p)$, ef $f_{1}=\left\langle K_{i} i n(b, p), K_{j} i n(b, p)\right\rangle$, and ef $f_{2}=\left\langle K_{i} \neg i n(b, p), K_{j} \neg i n(b, p)\right\rangle$.

Actually, when $K_{i} i n(b, p)$, tell $(i, j, b, p)$ should result in common knowledge of $i n(b, p)$ between the two agents. Since we do not support arbitrary common knowledge, we have to approximate common knowledge with higher-order knowledge. For example, we can express the conditional effect with $K_{j} i n(b, p) \wedge K_{i} K_{j} i n(b, p) \wedge K_{j} K_{i} i n(b, p) \wedge$ $K_{i} K_{j} K_{i} i n(b, p) \wedge K_{j} K_{i} K_{j} i n(b, p)$.

Definition 3.3 A sensing action is a triple $\langle$ pre, pos, neg $\rangle$ of $\mathcal{L}_{K}$ formulas, where pre, pos, and neg indicate the precondition, the positive result, and the negative result, respectively.

For the example of $\operatorname{find}(i, b, p)$, pre $=a t(i, p) \wedge$ $K_{i} a t(i, p)$, pos $=i n(b, p) \wedge K_{i} i n(b, p)$, and neg $=$ $\neg i n(b, p) \wedge K_{i} \neg i n(b, p)$.

An action $a$ is executable wrt a $\mathrm{KB} \phi \in \mathcal{L}_{K}$ if $\phi \models_{\gamma}$ pre (a). Suppose $a$ is executable wrt $\phi$. The progression of $\phi$ wrt $a$ is defined by resorting to a revision operator $\circ_{\gamma}$ and an update operator $\diamond_{\gamma}$ for $\mathcal{L}_{K}$ : we use update for deterministic actions and revision for sensing actions.

Definition 3.4 Let $\phi \in \mathcal{L}_{K}$, and $a_{d}$ a deterministic action where eff $f\left(a_{d}\right)=\left\{\left\langle c_{1}, e_{1}\right\rangle, \ldots,\left\langle c_{n}, e_{n}\right\rangle\right\}$. Let $c_{i_{1}}, \ldots, c_{i_{m}}$ be all the $c_{i}$ 's s.t. $\phi \models_{\gamma} c_{i}$. Then $\phi^{\prime}$, denoted by $\operatorname{prog}\left(\phi, a_{d}\right)$, is a progression of $\phi$ wrt $a_{d}$ if $\phi^{\prime}=\left(\left(\phi \diamond_{\gamma} e_{i_{1}}\right) \ldots\right) \diamond_{\gamma} e_{i_{m}}$.

Definition 3.5 Let $\phi \in \mathcal{L}_{K}$, and $a_{s}$ a sensing action. Then a pair $\left(\phi^{+}, \phi^{-}\right)$, written $\operatorname{prog}\left(\phi, a_{s}\right)$, is a progression of $\phi$ wrt $a_{s}$ if $\phi^{+}=\phi \circ_{\gamma} \operatorname{pos}\left(a_{s}\right)$, and $\phi^{-}=\phi \circ_{\gamma} n e g\left(a_{s}\right)$.

A solution of a MEP problem is an action tree branching on sensing results.

Definition 3.6 Let $\mathcal{Q}$ be a MEP problem $\langle\mathcal{A}, \mathcal{P}, \mathcal{D}, \mathcal{S}, \mathcal{I}, \mathcal{G}, \gamma\rangle$. The set $\mathcal{T}$ of action trees is inductively defined:

1. $\epsilon$ is in $\mathcal{T}$, here $\epsilon$ represents the empty tree;

2. if $a_{d} \in \mathcal{D}$ and $T \in \mathcal{T}$, then $a_{d} ; T$ is in $\mathcal{T}$;

3. if $a_{s} \in \mathcal{S}, T^{+}, T^{-} \in \mathcal{T}$, then $a_{s} ;\left(T^{+} \mid T^{-}\right)$is in $\mathcal{T}$.

Definition 3.7 Let $\phi \in \mathcal{L}_{K}, T$ an action tree. The progression of $\phi$ wrt $T$, written $\operatorname{prog}(\phi, T)$, is inductively defined:

1. $\operatorname{prog}(\phi, \epsilon)=\{\phi\}$

2. if $\phi \models_{\gamma} \operatorname{pre}\left(a_{d}\right), \operatorname{prog}\left(\phi, a_{d} ; T^{\prime}\right)=\operatorname{prog}\left(\phi^{\prime}, T^{\prime}\right)$, where $\operatorname{prog}\left(\phi, a_{d}\right)=\phi^{\prime}$

3. if $\phi \models_{\gamma} \operatorname{pre}\left(a_{s}\right), \operatorname{prog}\left(\phi, a_{s} ;\left(T^{+} \mid T^{-}\right)\right)=\operatorname{prog}\left(\phi^{+}, T^{+}\right)$ $\cup \operatorname{prog}\left(\phi^{-}, T^{-}\right)$, where $\operatorname{prog}\left(\phi, a_{s}\right)=\left(\phi^{+}, \phi^{-}\right)$;

4. otherwise, $\operatorname{prog}(\phi, T)$ is undefined.

Definition 3.8 Let $\mathcal{Q}$ be a MEP problem $\langle\mathcal{A}, \mathcal{P}, \mathcal{D}, \mathcal{S}, \mathcal{I}, \mathcal{G}, \gamma\rangle$. An action tree $T$ is a solution of $\mathcal{Q}$ if $\operatorname{prog}(\mathcal{I}, T)$ is defined, and for each formula $\phi$ in $\operatorname{prog}(\mathcal{I}, T), \phi \models_{\gamma} \mathcal{G}$.

Figure 1 shows a solution for Example 1. First, Agent 1 moves left. Then she senses if $b_{1}$ is in $p_{1}$. If $b_{1}$ is in $p_{1}$, agent 2 moves right, and senses if $b_{2}$ is in $p_{3}$. If $b_{1}$ is not in $p_{1}$, agent 1 senses if $b_{2}$ is in $p_{1}$, and tells the result to agent 2 . 


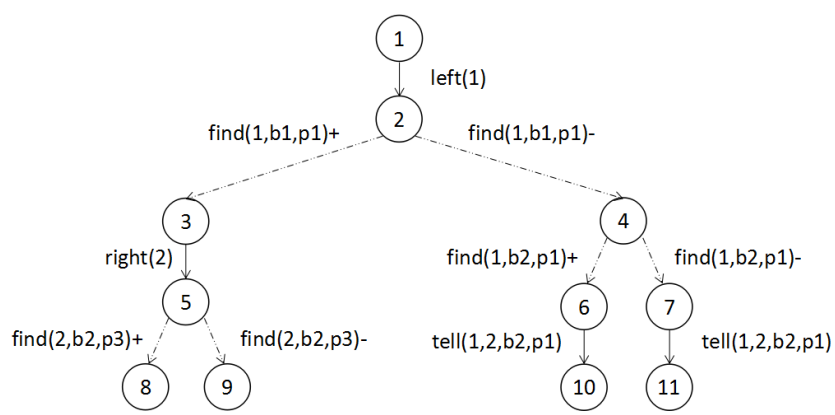

Figure 1: A solution to Example 1

\section{Our Algorithms}

In this section, we present our reasoning, belief change, and planning algorithms.

To support efficient reasoning and belief change, we represent KBs as ACDFs, queries as the negation of ACDFs, revision or update formulas as ACDFs, and constraints as DNFs. Thus, for a planning problem, our planner first compiles the initial $\mathrm{KB}$, the effects of conditional effects of deterministic actions, and the positive and negative results of sensing actions into ACDFs, the preconditions of actions, the conditions of conditional effects, and the goal into the negation of ACDFs, and finally the constraint into DNF.

For Example 1, after compilation, we get

- $\mathcal{I}=a t\left(1, p_{2}\right) \wedge a t\left(2, p_{2}\right) \wedge \neg i n\left(b_{1}, p_{2}\right) \wedge \neg i n\left(b_{2}, p_{2}\right) \wedge$ $\nabla_{1}\left\{a t\left(1, p_{2}\right) \wedge \neg i n\left(b_{1}, p_{2}\right) \wedge \neg i n\left(b_{2}, p_{2}\right)\right\} \wedge$

$\nabla_{2}\left\{a t\left(2, p_{2}\right) \wedge \neg i n\left(b_{1}, p_{2}\right) \wedge \neg i n\left(b_{2}, p_{2}\right)\right\}$;

- $\operatorname{pre}(\operatorname{left}(i))=\neg\left(\operatorname{at}\left(i, p_{1}\right) \vee \nabla_{i}\left\{\top, \operatorname{at}\left(i, p_{1}\right)\right\}\right)$;

- $\operatorname{cef}\left(\operatorname{eff} f_{1}(\operatorname{left}(i))\right)=\operatorname{at}\left(i, p_{1}\right) \wedge \nabla_{i}\left\{a t\left(i, p_{1}\right)\right\}$;

- $\operatorname{pos}(\operatorname{find}(i, b, p))=i n(b, p) \wedge \nabla_{i}\{i n(b, p)\}$;

- $\mathcal{G}=\neg \bigvee_{i=1}^{2} \nabla_{i}\left\{\top, \neg i n\left(b_{i}, p_{1}\right), \neg i n\left(b_{i}, p_{2}\right), \neg i n\left(b_{i}, p_{3}\right)\right\}$;

- $\gamma$ is a DNF of $3^{4}$ terms.

\subsection{Strong Entailment and Equivalence}

Our planner searches through the space of KBs, represented as ACDFs, and performs loop detection during search. Unfortunately, it is not tractable to check the equivalence of two ACDFs. Thus we introduce a stronger notion of equivalence which can be checked in polynomial time.

Definition 4.1 Let $\phi$ and $\phi^{\prime}$ be two ACDFs, $\gamma$ a DNF. The strong entailment relation $\phi \mapsto_{\gamma} \phi^{\prime}$ is recursively defined:

1. For propositional terms $\phi$ and $\phi^{\prime}, \phi \mapsto_{\gamma} \phi^{\prime}$ if $\phi \wedge \gamma \models \phi^{\prime}$.

2. When $\phi=\phi_{0} \wedge \bigwedge_{a \in \mathcal{A}} \nabla_{a} \Phi_{a}$ and $\phi^{\prime}=\phi_{0}^{\prime} \wedge \bigwedge_{a \in \mathcal{A}} \nabla_{a} \Phi_{a}^{\prime}$, $\phi \mapsto_{\gamma} \phi^{\prime}$ if the following hold:

(a) $\phi_{0} \mapsto_{\gamma} \phi_{0}^{\prime}$;

(b) for each $a \in \mathcal{A}$, for each $\phi_{a} \in \Phi_{a}$ there exists $\phi_{a}^{\prime} \in \Phi_{a}^{\prime}$ s.t. $\phi_{a} \mapsto_{\gamma} \phi_{a}^{\prime}$;

(c) for each $a \in \mathcal{A}$, for each $\phi_{a}^{\prime} \in \Phi_{a}^{\prime}$ there exists $\phi_{a} \in \Phi_{a}$ s.t. $\phi_{a} \mapsto_{\gamma} \phi_{a}^{\prime}$.

3. When $\phi=\bigvee \Phi$ and $\phi^{\prime}=\bigvee \Phi^{\prime}, \phi \mapsto_{\gamma} \phi^{\prime}$ if for all $\phi \in \Phi$ there exists $\phi^{\prime} \in \Phi^{\prime}$ s.t. $\phi \mapsto_{\gamma} \phi^{\prime}$.
Clearly, the strong entailment relation can be checked in polynomial time. By Proposition 2.12, it is easy to prove:

Proposition 4.2 Let $\phi$ and $\phi^{\prime}$ be two ACDFs, and $\gamma$ a DNF. If $\phi \mapsto_{\gamma} \phi^{\prime}$, then $\phi=_{\gamma} \phi^{\prime}$.

We say that two ACDFs $\phi$ and $\phi^{\prime}$ are strongly equivalent wrt constraint $\gamma$ if both $\phi \mapsto_{\gamma} \phi^{\prime}$ and $\phi^{\prime} \mapsto_{\gamma} \phi$.

\subsection{Reasoning}

As mentioned earlier, to support efficient reasoning, we represent KBs as ACDFs, and queries as the negation of ACDFs. By Propositions 2.6 and 2.13, it is easy to prove

Proposition 4.3 Let $\phi$ and $\phi^{\prime}$ be two ACDFs, $\gamma$ a DNF.

1. If $\phi$ and $\phi^{\prime}$ are propositional terms, $\phi \models_{\gamma} \neg \phi^{\prime}$ iff for each term $t$ in $\gamma, \phi \wedge t \wedge \phi^{\prime}$ has complementary literals.

2. If $\phi=\phi_{0} \wedge \bigwedge_{a \in \mathcal{A}} \nabla_{a} \Phi_{a}$ and $\phi^{\prime}=\phi_{0}^{\prime} \wedge \bigwedge_{a \in \mathcal{A}} \nabla_{a} \Phi_{a}^{\prime}$, then $\phi \models_{\gamma} \neg \phi^{\prime}$ iff one of the following holds:

(a) $\phi_{0} \models{ }_{\gamma} \neg \phi_{0}^{\prime}$;

(b) there exist $a \in \mathcal{A}$ and $\phi \in \Phi_{a}$ s.t. for all $\phi^{\prime} \in \Phi_{a}^{\prime}$, $\phi \models \gamma \neg \phi^{\prime}$;

(c) there exist $a \in \mathcal{A}$ and $\phi^{\prime} \in \Phi_{a}^{\prime}$ s.t. for all $\phi \in \Phi_{a}$, $\phi \models_{\gamma} \neg \phi^{\prime}$.

3. If $\phi=\bigvee \Phi$ and $\phi^{\prime}=\bigvee \Phi^{\prime}$, then $\phi \models_{\gamma} \neg \phi^{\prime}$ iff for all $\phi \in \Phi$ and $\phi^{\prime} \in \Phi^{\prime},\left.\phi\right|_{\gamma} \neg \phi^{\prime}$.

The above proposition gives us a recursive algorithm for deciding if $\phi \models_{\gamma} \neg \phi^{\prime}$, or equivalently, if $\phi \wedge \phi^{\prime}$ is satisfiable wrt $\gamma$. Clearly, the algorithm has polynomial complexity.

For Example 1, to check if $\mathcal{I} \models_{\gamma} \operatorname{pre}(\operatorname{left}(1))$, by Rule 3, we check if both $\mathcal{I} \equiv_{\gamma} \neg a t\left(1, p_{1}\right)$ and $\mathcal{I} \models_{\gamma}$ $\neg \nabla_{1}\left\{\top\right.$, at $\left.\left(1, p_{1}\right)\right\}$ hold. By Rule 1 , the former holds. By Rule 2.a, the latter holds, since $a t\left(1, p_{2}\right) \models_{\gamma} \neg a t\left(1, p_{1}\right)$.

\subsection{Higher-order Belief Change}

The basic idea behind our belief change operators is to reduce change of epistemic formulas to that of lower-order epistemic formulas, and as basis we resort to change of propositional formulas. The essential difference between revision and update is: revision satisfies the conjunction property that when $\phi \wedge \phi^{\prime}$ is satisfiable, $\phi \circ \phi^{\prime} \Leftrightarrow \phi \wedge \phi^{\prime}$, while update satisfies the distribution property that when both $\phi_{1}$ and $\phi_{2}$ are satisfiable, $\left(\phi_{1} \vee \phi_{2}\right) \diamond \phi^{\prime} \Leftrightarrow\left(\phi_{1} \diamond \phi^{\prime} \vee \phi_{2} \diamond \phi^{\prime}\right)$.

We begin with some notation. If there exist $\phi \in \Phi$ and $\phi^{\prime} \in$ $\Phi^{\prime}$ s.t. $\phi \wedge \phi^{\prime}$ is satisfiable wrt $\gamma$, we let $\Phi *_{\gamma} \Phi^{\prime}=\left\{\left(\phi, \phi^{\prime}\right) \mid\right.$ $\phi \in \Phi, \phi^{\prime} \in \Phi^{\prime}, \phi \wedge \phi^{\prime}$ is satisfiable wrt $\left.\gamma\right\}$; otherwise, let $\Phi *_{\gamma} \Phi^{\prime}=\left\{\left(\phi, \phi^{\prime}\right) \mid \phi \in \Phi, \phi^{\prime} \in \Phi^{\prime}\right\}$. Intuitively, whenever possible, $\Phi *_{\gamma} \Phi^{\prime}$ restricts our attention to those consistent pairs of formulas. We let $\Phi \circ \phi^{\prime}$ denote the set $\left\{\phi \circ \phi^{\prime} \mid \phi \in\right.$ $\Phi\}$, and similarly for $\diamond$.

Definition 4.4 Let $\phi$ and $\phi^{\prime}$ be ACDFs, $\gamma$ a DNF. The revision of $\phi$ with $\phi^{\prime}$ under $\gamma, \phi \circ_{\gamma} \phi^{\prime}$, is recursively defined:

1. When $\phi$ and $\phi^{\prime}$ are propositional, the result is $\phi \circ_{s}\left(\phi^{\prime} \wedge\right.$ $\gamma)$, where $\circ_{s}$ is Satoh's revision operator.

2. When $\phi=\phi_{0} \wedge \bigwedge_{a \in \mathcal{B}} \nabla_{a} \Phi_{a}, \phi^{\prime}=\phi_{0}^{\prime} \wedge \bigwedge_{a \in \mathcal{B}^{\prime}} \nabla_{a} \Phi_{a}^{\prime}$, $\phi \wedge \phi^{\prime}$ is satisfiable wrt $\gamma, \phi \circ_{\gamma} \phi^{\prime}$ is defined as:

$$
\begin{aligned}
& \left(\phi_{0} \circ_{\gamma} \phi_{0}^{\prime}\right) \wedge \bigwedge a \in \mathcal{B}-\mathcal{B}^{\prime} \nabla_{a} \Phi_{a} \wedge \bigwedge_{a \in \mathcal{B}^{\prime}-\mathcal{B}} \nabla_{a} \Phi_{a}^{\prime} \wedge \\
& \bigwedge a \in{\mathcal{B} \cap \mathcal{B}^{\prime}} \nabla_{a}\left[\left(\Phi_{a} \circ_{\gamma} \bigvee \Phi_{a}^{\prime}\right) \cup\left(\Phi_{a}^{\prime} \circ_{\gamma} \bigvee \Phi_{a}\right)\right] .
\end{aligned}
$$


3. When $\phi=\phi_{0} \wedge \bigwedge_{a \in \mathcal{B}} \nabla_{a} \Phi_{a}, \phi^{\prime}=\phi_{0}^{\prime} \wedge \bigwedge_{a \in \mathcal{B}^{\prime}} \nabla_{a} \Phi_{a}^{\prime}$, $\phi \wedge \phi^{\prime}$ is unsatisfiable wrt $\gamma, \phi \circ_{\gamma} \phi^{\prime}$ is defined as:

$$
\begin{aligned}
\left(\phi_{0} \circ_{\gamma} \phi_{0}^{\prime}\right) \wedge & \bigwedge_{a \in \mathcal{B}-\mathcal{B}^{\prime}} \nabla_{a} \Phi_{a} \wedge \bigwedge_{a \in \mathcal{B}^{\prime}-\mathcal{B}} \nabla_{a} \Phi_{a}^{\prime} \wedge \\
& \bigwedge_{a \in \mathcal{B} \cap \mathcal{B}^{\prime}} \nabla_{a}\left[\Phi_{a}^{*} \cup\left(\Phi_{a}^{\prime}-\Phi_{a}^{\prime \prime}\right)\right],
\end{aligned}
$$

where $\Phi_{a}^{*}=\left\{\phi \circ_{\gamma} \phi^{\prime} \mid\left(\phi, \phi^{\prime}\right) \in \Phi_{a} *_{\gamma}\left\{\bigvee \Phi_{a}^{\prime}\right\}\right\}, \Phi_{a}^{\prime \prime}=$ $\left\{\phi^{\prime} \in \Phi_{a}^{\prime} \mid\right.$ there exists a $\phi \in \Phi_{a}^{*}$ s.t. $\left.\phi \mid=_{\gamma} \neg\left(\neg \phi^{\prime}\right)\right\}$, where $\neg \phi^{\prime}$ is converted to ACDF during compilation.

4. $(\bigvee \Phi) \circ_{\gamma}\left(\bigvee \Phi^{\prime}\right)=\bigvee\left\{\phi \circ_{\gamma} \phi^{\prime} \mid\left(\phi, \phi^{\prime}\right) \in \Phi *_{\gamma} \Phi^{\prime}\right\}$.

Rule 2 and 4 are for the purpose of the conjunction property. Recall that $\nabla_{a} \Phi_{a} \Leftrightarrow K_{a}(\bigvee \Phi) \wedge L_{a} \Phi$, hence $\bigvee \Phi_{a}$ is the belief of agent $a$, and each $\phi \in \Phi_{a}$ is a possibility for agent $a$. The intuition behind Rule 3 is as follows. When there exist old possibilities that are consistent with the new belief $\bigvee \Phi_{a}^{\prime}$, we just keep these possibilities, otherwise we revise each possibility with the new belief, which explains the definition of $\Phi_{a}^{*}$. Also, among all the new possibilities, we remove those weaker ones, getting $\Phi_{a}^{\prime}-\Phi_{a}^{\prime \prime}$. This is because we would like to get a revision result as strong as possible. For example, suppose $\Phi_{a}^{*}=\{p \wedge q\}$, and $\Phi_{a}^{\prime}=\{p\}$; then we obtain $\nabla_{a}\{p \wedge q\}$, which is strictly stronger than $\nabla_{a}\{p \wedge q, p\}$.

Note that except for a polynomial number of calls to propositional revision, the revision operation can be done in polynomial time.

We say that an ACDF is disjunct-wise satisfiable (dsatisfiable for short) wrt $\gamma$ if it is a disjunctive CDF and each disjunct is satisfiable wrt $\gamma$, or it is a propositional or CDF term which is satisfiable wrt $\gamma$.

Proposition 4.5 Let $\phi$ and $\phi^{\prime}$ be ACDFs d-satisfiable wrt $\gamma$. Let $\phi^{*}=\phi \circ_{\gamma} \phi^{\prime}$. Then $\phi^{*}$ is d-satisfiable wrt $\gamma$, and $\phi^{*} \models_{\gamma}$ $\phi^{\prime}$. Moreover, when $\phi \wedge \phi^{\prime}$ is satisfiable wrt $\gamma, \phi^{*} \Leftrightarrow_{\gamma} \phi \wedge \phi^{\prime}$.

Proof: We prove by induction on $\|\phi\|+\left\|\phi^{\prime}\right\|$.

1. $\phi$ and $\phi^{\prime}$ are propositional. The properties follow from the definition of Satoh's revision.

2. $\phi=\phi_{0} \wedge \bigwedge_{a \in \mathcal{B}} \nabla_{a} \Phi_{a}, \phi^{\prime}=\phi_{0}^{\prime} \wedge \bigwedge_{a \in \mathcal{B}^{\prime}} \nabla_{a} \Phi_{a}^{\prime}, \phi \wedge \phi^{\prime}$ is satisfiable wrt $\gamma$. Let $a \in \mathcal{B} \cap \mathcal{B}^{\prime}$. Since $\phi \wedge \phi^{\prime}$ is satisfiable wrt $\gamma$, by Proposition 2.11, for each $\phi_{a} \in \Phi_{a}, \phi_{a} \wedge \bigvee \Phi_{a}^{\prime}$ is satisfiable wrt $\gamma$. By induction, $\phi_{a} \circ \gamma \bigvee \Phi_{a}^{\prime} \Leftrightarrow \gamma \phi_{a} \wedge \bigvee^{a} \Phi_{a}^{\prime}$. Similarly, for each $\phi_{a}^{\prime} \in \Phi_{a}^{\prime}, \phi_{a}^{\prime} \circ_{\gamma} \bigvee \Phi_{a} \Leftrightarrow \gamma \phi_{a}^{\prime} \wedge \bigvee \Phi_{a}$. By Proposition 2.6 (3), $\phi \circ_{\gamma} \phi^{\prime} \Leftrightarrow_{\gamma} \phi \wedge \phi^{\prime}$. It follows that $\phi \circ_{\gamma} \phi^{\prime}$ is satisfiable wrt $\gamma$ and $\phi \circ_{\gamma} \phi^{\prime} \models_{\gamma} \phi^{\prime}$.

3. $\phi=\phi_{0} \wedge \bigwedge_{a \in \mathcal{B}} \nabla_{a} \Phi_{a}, \phi^{\prime}=\phi_{0}^{\prime} \wedge \bigwedge_{a \in \mathcal{B}^{\prime}} \nabla_{a} \Phi_{a}^{\prime}, \phi \wedge \phi^{\prime}$ is unsatisfiable wrt $\gamma$. Let $a \in \mathcal{B} \cap \mathcal{B}^{\prime}$. By induction, for each $\phi_{a} \in \Phi_{a}, \phi_{a} \circ_{\gamma} \bigvee \Phi_{a}^{\prime}$ is d-satisfiable wrt $\gamma$, hence each element of $\Phi_{a}^{*}$ is d-satisfiable wrt $\gamma$. Thus $\phi \circ_{\gamma} \phi^{\prime}$ is satisfiable wrt $\gamma$. By induction, $\phi_{0} \circ \gamma \phi_{0}^{\prime} \models_{\gamma} \phi_{0}^{\prime}$, and for each $\phi_{a}^{*} \in \Phi_{a}^{*}$, $\phi_{a}^{*} \models_{\gamma} \bigvee \Phi_{a}^{\prime}$. Also, by the definition of $\Phi_{a}^{\prime \prime}$, for each $\phi_{a}^{\prime} \in$ $\Phi_{a}^{\prime}$, there exists $\phi_{a}^{*} \in \Phi_{a}^{*} \cup\left(\Phi_{a}^{\prime}-\Phi_{a}^{\prime \prime}\right)$ s.t. $\phi_{a}^{*} \models_{\gamma} \phi_{a}^{\prime}$. By Proposition 2.12, $\phi \circ_{\gamma} \phi^{\prime} \models_{\gamma} \phi^{\prime}$.

4. $\phi=\bigvee \Phi, \phi^{\prime}=\bigvee \Phi^{\prime}, \phi \wedge \phi^{\prime}$ is satisfiable wrt $\gamma$. Then $\Phi *_{\gamma} \Phi^{\prime}=\left\{\left(\phi_{1}, \phi_{1}^{\prime}\right) \mid \phi_{1} \in \Phi_{1}, \phi_{1}^{\prime} \in \Phi_{1}^{\prime}, \phi_{1} \wedge \phi_{1}^{\prime}\right.$ is satisfiable wrt $\gamma\}$. By induction, for each $\left(\phi_{1}, \phi_{1}^{\prime}\right) \in \Phi *_{\gamma} \Phi^{\prime}, \phi_{1} \circ_{\gamma} \phi_{1}^{\prime}$ is satisfiable wrt $\gamma$, and $\phi_{1} \circ_{\gamma} \phi_{1}^{\prime} \Leftrightarrow \gamma \phi_{1} \wedge \phi_{1}^{\prime}$. Thus $\phi \circ_{\gamma} \phi^{\prime}$ is d-satisfiable wrt $\gamma$, and $\phi \circ_{\gamma} \phi^{\prime}=\bigvee\left\{\phi_{1} \circ_{\gamma} \phi_{1}^{\prime} \mid\left(\phi_{1}, \phi_{1}^{\prime}\right) \in\right.$ $\left.\Phi *_{\gamma} \Phi^{\prime}\right\} \Leftrightarrow_{\gamma} \bigvee\left\{\phi_{1} \wedge \phi_{1}^{\prime} \mid\left(\phi_{1}, \phi_{1}^{\prime}\right) \in \Phi *_{\gamma} \Phi^{\prime}\right\} \Leftrightarrow_{\gamma} \phi \wedge \phi^{\prime}$. It follows that $\phi \circ_{\gamma} \phi^{\prime} \models_{\gamma} \phi^{\prime}$.
5. $\phi=\bigvee \Phi, \phi^{\prime}=\bigvee \Phi^{\prime}, \phi \wedge \phi^{\prime}$ is unsatisfiable wrt $\gamma$. Then $\Phi *_{\gamma} \Phi^{\prime}=\left\{\left(\phi_{1}, \phi_{1}^{\prime}\right) \mid \phi_{1} \in \Phi_{1}, \phi_{1}^{\prime} \in \Phi_{1}^{\prime}\right\}$. Since both $\phi$ and $\phi^{\prime}$ are d-satisfiable wrt $\gamma$, for each $\left(\phi_{1}, \phi_{1}^{\prime}\right) \in \Phi *_{\gamma} \Phi^{\prime}$, both $\phi_{1}$ and $\phi_{1}^{\prime}$ are satisfiable wrt $\gamma$. By induction, $\phi_{1} \circ_{\gamma} \phi_{1}^{\prime}$ is satisfiable wrt $\gamma$, and $\phi_{1} \circ_{\gamma} \phi_{1}^{\prime} F_{\gamma} \phi_{1}^{\prime}$. Hence $\phi \circ_{\gamma} \phi^{\prime}$ is d-satisfiable wrt $\gamma$, and $\phi \circ_{\gamma} \phi^{\prime} \models \phi^{\prime}$.

Definition 4.6 Let $\phi$ and $\phi^{\prime}$ be ACDFs, $\gamma$ a DNF. The update of $\phi$ with $\phi^{\prime}$ under $\gamma$, denoted $\phi \diamond_{\gamma} \phi^{\prime}$, is recursively defined:

1. When $\phi$ and $\phi^{\prime}$ are propositional, the result is $\phi \diamond_{w}\left(\phi^{\prime} \wedge\right.$ $\gamma)$, where $\diamond_{w}$ is Winslett's update operator.

2. When $\phi=\phi_{0} \wedge \bigwedge_{a \in \mathcal{B}} \nabla_{a} \Phi_{a}$ and $\phi^{\prime}=\phi_{0}^{\prime} \wedge \bigwedge_{a \in \mathcal{B}^{\prime}} \nabla_{a} \Phi_{a}^{\prime}$, $\phi \diamond_{\gamma} \phi^{\prime}$ is defined as follows:

$$
\begin{aligned}
& \left(\phi_{0} \diamond_{\gamma} \phi_{0}^{\prime}\right) \wedge \bigwedge a \in \mathcal{B}-\mathcal{B}^{\prime} \nabla_{a} \Phi_{a} \wedge \bigwedge_{a \in \mathcal{B}^{\prime}-\mathcal{B}} \nabla_{a} \Phi_{a}^{\prime} \wedge \\
& \bigwedge a \in{\mathcal{B} \cap \mathcal{B}^{\prime}} \nabla_{a}\left[\Phi_{a}^{*} \cup\left(\Phi_{a}^{\prime}-\Phi_{a}^{\prime \prime}\right)\right],
\end{aligned}
$$

where $\Phi_{a}^{*}=\Phi_{a} \diamond_{\gamma} \bigvee \Phi_{a}^{\prime}, \Phi_{a}^{\prime \prime}=$ $\left\{\phi^{\prime} \in \Phi_{a}^{\prime} \mid\right.$ there exists a $\phi \in \Phi_{a}^{*}$ s.t. $\left.\phi \models_{\gamma} \neg\left(\neg \phi^{\prime}\right)\right\}$, where $\neg \phi^{\prime}$ is converted to ACDF during compilation.

3. $(\bigvee \Phi) \diamond_{\gamma} \phi^{\prime}=\bigvee_{\phi \in \Phi} \phi \diamond_{\gamma} \phi^{\prime}$.

4. When $\phi$ is a CDF term, $\phi \diamond_{\gamma}\left(\bigvee \Phi^{\prime}\right)=\bigvee\left\{\phi \diamond_{\gamma} \phi^{\prime} \mid\right.$ $\left.\left(\phi, \phi^{\prime}\right) \in\{\phi\} *_{\gamma} \Phi^{\prime}\right\}$.

Rule 3 and the definition of $\Phi_{a}^{*}$ in Rule 2 are for the purpose of the distribution property. Rule 4 is to approximate the principle of minimal change: when there is $\phi^{\prime} \in \Phi^{\prime}$ s.t. $\phi^{\prime}$ is consistent with $\phi$, we simply ignore those which are not.

Note that except for a polynomial number of calls to propositional update, the update operation can be done in polynomial time.

Proposition 4.7 Let $\phi$ and $\phi^{\prime}$ be ACDFs d-satisfiable wrt $\gamma$. Let $\phi^{*}=\phi \diamond_{\gamma} \phi^{\prime}$. Then $\phi^{*}$ is d-satisfiable wrt $\gamma$, and $\phi^{*} \models_{\gamma}$ $\phi^{\prime}$. Moreover, $\left(\phi_{1} \vee \phi_{2}\right) \diamond_{\gamma} \phi^{\prime} \Leftrightarrow_{\gamma}\left(\phi_{1} \diamond_{\gamma} \phi^{\prime} \vee \phi_{2} \diamond_{\gamma} \phi^{\prime}\right)$.

Proof: We prove by induction on $\|\phi\|+\left\|\phi^{\prime}\right\|$.

1. $\phi$ and $\phi^{\prime}$ are propositional. The properties follow from the definition of Winslett's update.

2. $\phi=\phi_{0} \wedge \bigwedge_{a \in \mathcal{B}} \nabla_{a} \Phi_{a}, \phi^{\prime}=\phi_{0}^{\prime} \wedge \bigwedge_{a \in \mathcal{B}^{\prime}} \nabla_{a} \Phi_{a}^{\prime}$. Let $a \in \mathcal{B} \cap \mathcal{B}^{\prime}$. By induction, for each $\phi_{a} \in \Phi_{a}, \phi_{a} \diamond_{\gamma} \bigvee \Phi_{a}^{\prime}$ is d-satisfiable wrt $\gamma$, hence each element of $\Phi_{a}^{*}$ is d-satisfiable wrt $\gamma$. Thus $\phi \diamond_{\gamma} \phi^{\prime}$ is satisfiable wrt $\gamma$. By induction, $\phi_{0} \diamond_{\gamma}$ $\phi_{0}^{\prime} \models_{\gamma} \phi_{0}^{\prime}$, and for each $\phi_{a}^{*} \in \Phi_{a}^{*}, \phi_{a}^{*} \models_{\gamma} \bigvee \Phi_{a}^{\prime}$. Also, by the definition of $\Phi_{a}^{\prime \prime}$, for each $\phi_{a}^{\prime} \in \Phi_{a}^{\prime}$, there exists $\phi_{a}^{*} \in \Phi_{a}^{*} \cup$ $\left(\Phi_{a}^{\prime}-\Phi_{a}^{\prime \prime}\right)$ s.t. $\phi_{a}^{*} \models_{\gamma} \phi_{a}^{\prime}$. By Proposition 2.12, $\phi \diamond_{\gamma} \phi^{\prime} \models_{\gamma} \phi^{\prime}$.

3. $\phi=\bigvee \Phi$. Clearly, the disjunction property holds. Since $\phi$ is d-satisfiable (wrt $\gamma$ ), each $\phi_{1} \in \Phi$ is satisfiable. By induction, for each $\phi_{1} \in \Phi, \phi_{1} \diamond_{\gamma} \phi^{\prime}$ is d-satisfiable and $\phi_{1} \diamond_{\gamma} \phi^{\prime} \models_{\gamma}$ $\phi^{\prime}$. Thus $\phi \diamond_{\gamma} \phi^{\prime}$ is d-satisfiable wrt $\gamma$ and $\phi \diamond_{\gamma} \phi^{\prime} \models_{\gamma} \phi^{\prime}$.

4. $\phi$ is a CDF term, $\phi^{\prime}=\bigvee \Phi^{\prime}$. Since $\phi^{\prime}$ is d-satisfiable (wrt $\gamma$ ), each $\phi_{1}^{\prime} \in \Phi^{\prime}$ is satisfiable. By induction, for each $\phi_{1}^{\prime} \in \Phi^{\prime}, \phi \diamond_{\gamma} \phi_{1}^{\prime}$ is d-satisfiable and $\phi \diamond_{\gamma} \phi_{1}^{\prime} \models_{\gamma} \phi_{1}^{\prime}$. Thus $\phi \diamond_{\gamma} \phi^{\prime}$ is d-satisfiable wrt $\gamma$ and $\phi \diamond_{\gamma} \phi^{\prime} \models_{\gamma} \phi^{\prime}$.

For Example 1, after doing action left(1), we get $\mathcal{I} \diamond_{\gamma} \operatorname{cef}\left(\right.$ ef $f_{1}($ left $(1))$, equivalent to $\phi_{1}$ under $\gamma$ : $\phi_{1}=\operatorname{at}\left(1, p_{1}\right) \wedge a t\left(2, p_{2}\right) \wedge \neg i n\left(b_{1}, p_{2}\right) \wedge \neg i n\left(b_{2}, p_{2}\right) \wedge$ $\nabla_{1}\left\{\operatorname{at}\left(1, p_{1}\right) \wedge \neg \operatorname{in}\left(b_{1}, p_{2}\right) \wedge \neg i n\left(b_{2}, p_{2}\right)\right\} \wedge$ 


$$
\nabla_{2}\left\{a t\left(2, p_{2}\right) \wedge \neg i n\left(b_{1}, p_{2}\right) \wedge \neg i n\left(b_{2}, p_{2}\right)\right\} .
$$

Now after doing find $\left(1, b_{1}, p_{1}\right)$ with a positive result, we get $\phi_{1} \circ_{\gamma} \operatorname{pos}\left(\operatorname{find}\left(1, b_{1}, p_{1}\right)\right)$, equivalent to $\phi_{2}$ under $\gamma$ :

$$
\begin{aligned}
\phi_{2}= & a t\left(1, p_{1}\right) \wedge a t\left(2, p_{2}\right) \wedge i n\left(b_{1}, p_{1}\right) \wedge \neg i n\left(b_{2}, p_{2}\right) \wedge \\
& \nabla_{1}\left\{a t\left(1, p_{1}\right) \wedge i n\left(b_{1}, p_{1}\right) \wedge \neg i n\left(b_{2}, p_{2}\right)\right\} \wedge \\
& \nabla_{2}\left\{a t\left(2, p_{2}\right) \wedge \neg \operatorname{in}\left(b_{1}, p_{2}\right) \wedge \neg i n\left(b_{2}, p_{2}\right)\right\} .
\end{aligned}
$$

\subsection{Planning}

Like [Wan et al., 2015], we adapt the PrAO algorithm for contingent planning [To et al., 2011] as our planning algorithm. PrAO extends AND/OR forward search with pruning techniques. It uses the so-called minimal DNF formulas to represent states and employs the following basic operations: reasoning, equivalence checking, update wrt ontic actions, and update wrt sensing actions. We use ACDFs to represent states, and for the above operations, we plug in our operations of reasoning, strong equivalence checking, progression wrt deterministic actions, and progression wrt sensing actions.

\section{Implementation and Experimentation}

Based on the theoretic work, we have developed EPDDL an extension of PDDL [McDermott et al., 1998], to describe multi-agent epistemic planning problems, and with naive implementations of Satoh's revision and Winslett's update operators, implemented a multi-agent epistemic planner MEPK. ${ }^{1}$

We evaluate MEPK with Selective-communication (SC) and Collaboration-and-communication (CC) domains adapted from [Kominis and Geffner, 2015], and Grapevine from [Muise et al., 2015], where SC is called "Corridor". We also made up three domains: Assembly-line (AL), and domains adapted from the classic Gossip problem [Attamah $e t$ al., 2014] and the knowledge game Hexa [van Ditmarsch, 2001]. We didn't consider three domains from [Kominis and Geffner, 2015]: MuddyChildren, Sum, and WordRooms. The first two involve public announcements, which result in common knowledge, and so we are not able to model them. The third is a variant of CC. Below we give description of each domain we use.

Selective-communication: $\mathrm{SC}(n)$. There are $n$ rooms in a corridor. The agents can move from a room to a neighboring room. When agent $i$ tells some information, all the other agents in the same room or in a neighboring room can hear what was told. Initially, each agent is in one of the rooms. The goal is that some agents get to know some information while some other agents do not. Versions of $\mathrm{SC}(\mathrm{n})$ with higher modal depth result from approximation of common knowledge with higher-order knowledge.

Collaboration-and-communication: $\mathrm{CC}(k, n),{ }^{*} \mathrm{CC}(k, n)$. These are variants of Example 1 where there are $k$ boxes and $n$ rooms. For efficiency reason, the $* \mathrm{CC}(k, n)$ variant adopt$\mathrm{s}$ an encoding somewhat different from that for Example 1. The $\mathrm{CC}(k, n)$ variant imitates the version from [Kominis and Geffner, 2015] in that with one action, an agent can see which boxes are in a room.

Grapevine $(n)$. A few guests attend a meeting in a villa with $n$ rooms. Each guest has his own secret to share with

\footnotetext{
${ }^{1}$ The link to our planner and domain sources is: https://github.com/sysulic/MEPK.
}

others. Each guest can move between the rooms, and broadcast his secret to the guests in the same room. The goal is that only some of the guests obtain the designated secrets.

Hexa Game. There are $k$ agents and $k$ cards, each with a unique color. Initially, everyone is holding a card, and can only see the color of his own card. A player can ask a question to another player whether his card is of a certain color. The question should always be honestly answered. The goal is for some agent to know the cards of all players.

Assembly-line (AL). There are two agents, each responsible for processing a part of a product. It is possible that an agent fails in processing his part. An agent can inform the other agent the status of his task. Two agents decide to assemble the product or restart depending on their knowledge of the status of the agents' tasks.

Gossip. Each of several friends has his own secret to share. Instead of sharing in public, they are only allowed to make a call to each other. In each call, they exchange all the secrets they know. The goal is that everyone knows all the secrets of other friends.

\begin{tabular}{|c|c|c|c|c|c|}
\hline Problem & $|\mathcal{A}|$ & $|\mathcal{S}|+|\mathcal{D}|$ & $|\mathcal{P}|$ & $d$ & MEPK \\
\hline CC(2,4) & 2 & $0+18$ & 18 & 1 & $0.14-0.13(3 / 4)$ \\
CC(3,4) & 2 & $0+18$ & 20 & 1 & $1.83-1.81(3 / 4)$ \\
CC $(4,4)$ & 2 & $0+18$ & 24 & 1 & $76.63-76.56(3 / 4)$ \\
$*$ CC $(2,3)$ & 2 & $8+16$ & 12 & 1 & $3.74-3.73(4 / 12)$ \\
$*$ CC $(2,3)$ & 3 & $12+42$ & 15 & 1 & $12.54-12.53(5 / 16)$ \\
$* C C(2,4)$ & 2 & $12+20$ & 16 & 1 & $9.40-9.39(6 / 18)$ \\
\hline SC(4) & 3 & $1+3$ & 7 & 1 & $0.03-0.02(5 / 10)$ \\
SC(4) & 7 & $1+3$ & 11 & 1 & $13.88-0.05(5 / 10)$ \\
SC(4) & 3 & $1+3$ & 7 & 3 & $0.04-0.03(5 / 10)$ \\
SC(4) & 3 & $1+3$ & 7 & 4 & $0.07-0.05(5 / 10)$ \\
SC(8) & 3 & $1+3$ & 11 & 1 & $0.22-0.16(10 / 19)$ \\
\hline Grapevine(2) & 3 & $0+18$ & 9 & 2 & $0.02-0.01(2 / 3)$ \\
Grapevine(2) & 4 & $0+56$ & 12 & 1 & $0.09-0.06(2 / 3)$ \\
Grapevine(2) & 4 & $0+56$ & 12 & 2 & $0.14-0.10(2 / 3)$ \\
Grapevine(2) & 4 & $0+56$ & 12 & 3 & $0.19-0.14(2 / 3)$ \\
Grapevine(2) & 4 & $0+56$ & 12 & 4 & $0.34-0.24(2 / 3)$ \\
Grapevine(3) & 4 & $0+152$ & 16 & 1 & $1.29-0.92(2 / 3)$ \\
\hline Hexa Game & 3 & $18+0$ & 9 & 1 & $0.01-0.01(1 / 3)$ \\
& 4 & $48+0$ & 16 & 1 & $0.02-0.02(3 / 11)$ \\
& 5 & $100+0$ & 25 & 1 & $28.25-25.19(6 / 47)$ \\
\hline Assemble Line & 2 & $4+2$ & 4 & 2 & $0.01-0.01(5 / 12)$ \\
& 2 & $4+2$ & 4 & 3 & $0.02-0.02(5 / 12)$ \\
& 2 & $4+2$ & 4 & 4 & $0.03-0.03(5 / 12)$ \\
Gossip & 4 & $0+24$ & 4 & 2 & $4.01-3.44(4 / 5)$ \\
& 5 & $0+120$ & 5 & 2 & $14.02-9.50(4 / 5)$ \\
\hline
\end{tabular}

Table 1: Experimental results.

\begin{tabular}{|c|c|c|c|c|c|}
\hline Problems & $|\mathcal{A}|$ & $d$ & MEPK & K\&G & Muise et al. \\
\hline $\mathrm{CC}(2,4)$ & 2 & 1 & $0.14-0.13(3 / 4)$ & $0.05-0.01(8)$ & na \\
$\mathrm{CC}(3,4)$ & 2 & 1 & $1.83-1.81(3 / 4)$ & $27.87-0.04(8)$ & na \\
$\mathrm{CC}(4,4)$ & 2 & 1 & $76.63-76.56(3 / 4)$ & $2115.98-2.09(8)$ & na \\
\hline $\mathrm{SC}(4)$ & 3 & 1 & $0.03-0.02(5 / 10)$ & $0.01-0.01(9)$ & $0.08-0.01(5)$ \\
$\mathrm{SC}(4)$ & 7 & 1 & $13.88-0.05(5 / 10)$ & na & $0.20-0.01(5)$ \\
$\mathrm{SC}(4)$ & 3 & 3 & $0.04-0.03(5 / 10)$ & na & $0.37-0.01(5)$ \\
\hline Grapevine(2) & 3 & 2 & $0.02-0.01(2 / 3)$ & na & $0.50-0.01(5)$ \\
Grapevine(2) & 4 & 1 & $0.09-0.06(2 / 3)$ & na & $0.34-0.01(9)$ \\
Grapevine(2) & 4 & 2 & $0.14-0.10(2 / 3)$ & na & $1.82-0.29(5)$ \\
\hline
\end{tabular}

Table 2: Comparison results. 
Our experiments were run on a Linux machine with 2.50GHz CPU and 4GB RAM. Our experimental results are shown in Table 1 . The 2 nd-5th columns indicate the number of agents, the number of sensing and deterministic actions, the number of atoms, and the modal depth of the KBs. If the number of sensing actions is 0 , the planning problem is conformant, else it is contingent. In the MEPK column, A-B $(\mathrm{X} / \mathrm{Y})$ stands for $A$ seconds of total time, $B$ seconds spent on search, and depth $X$ and $Y$ nodes of solution tree. The results show the viability of our approach. Nonetheless, our planner does not scale well on Hexa. This is because the search performance is greatly influenced by the number of sensing actions. In Hexa, there are 48 (resp. 100) sensing actions when there are 4 (resp. 5) agents.

We reran the corresponding experiments of [Kominis and Geffner, 2015] and [Muise et al., 2015] using an FF planner and their domain sources, and the comparison results are shown in Table 2 . In the last two columns, $(X)$ stands for plan length $X$, and "na" means that the planning problem is not considered by the planner. For example, CC is not encoded by Muise et al. ; as to SC, K\&G only encodes SC(4) with 3 agents and $d=1$. The results show that the searching performance of MEPK is reasonable, but still worse than the two other planners. This is because MEPK uses more general and complex KBs, and hence spends more time on reasoning and progression. However, when it comes to total time, MEPK performs better than the two other planners, with the exception of SC(4) with 7 agents. This is because MEPK saves from the expensive compilation into classical planning.

\section{Conclusions}

In this paper, we have proposed a general representation framework for multi-agent epistemic planning where arbitrary multi-agent epistemic formulas are used and solutions are action trees. To support efficient reasoning and progression, we resort to alternating cover disjunctive formulas, and propose basic revision and update algorithms for them. Based on the theoretic work, we have implemented a native multiagent epistemic planner MEPK, which does not rely on compilation into classical planning. Experimental results have demonstrated the viability of our approach. In the future, we are interested in doing a model-theoretic study of higherorder belief change and improving our revision and update algorithms. Also, we would like to extend our current work to accommodate common knowledge.

\section{Acknowledgments}

We thank the anonymous reviewers for helpful comments. We are grateful to Liangda Fang for helpful discussions on the paper, and Hans van Ditmarsch for introducing us to the normal form of alternating cover disjunctive formulas. Xiao Huang and Yongmei Liu acknowledge support from the Natural Science Foundation of China under Grant Nos. 61572535 and 61463044. Biqing Fang and Hai Wan acknowledge support from National Natural Science Foundation of China under grant 61573386, and Natural Science Foundation of Guangdong Province under grant 2016A030313292.

\section{References}

[Alchourrón et al., 1985] Carlos E. Alchourrón, Peter Gärdenfors, and David Makinson. On the logic of theory change: Partial meet contraction and revision functions. The Journal of Symbolic Logic, 50(2):510-530, 1985.

[Attamah et al., 2014] Maduka Attamah, Hans van Ditmarsch, Davide Grossi, and Wiebe van der Hoek. Knowledge and gossip. In Proc. 21st European Conference on Artificial Intelligence (ECAI-2014), pages 21-26, 2014.

[Aucher and Bolander, 2013] Guillaume Aucher and Thomas Bolander. Undecidability in epistemic planning. In Proceedings of the 23rd International Joint Conference on Artificial Intelligence, pages 27-33, 2013.

[Aucher, 2010] Guillaume Aucher. Generalizing AGM to a multi-agent setting. Logic Journal of the IGPL, 18(4):530558, 2010.

[Aucher, 2011] Guillaume Aucher. DEL-sequents for progression. Journal of Applied Non-Classical Logics, 21(34):289-321, 2011.

[Bolander and Andersen, 2011] T. Bolander and M. B. Andersen. Epistemic planning for single and multi-agent systems. Journal of Applied Non-Classical Logics, 21(1):934, 2011.

[Caridroit et al., 2016] Thomas Caridroit, Sébastien Konieczny, Tiago de Lima, and Pierre Marquis. On distances between KD45n Kripke models and their use for belief revision. In ECAI 2016, pages 1053-1061, 2016.

[D'Agostino and Lenzi, 2006] G. D'Agostino and G. Lenzi. On modal $\mu$-calculus with explicit interpolants. Journal of Applied Logic, 4:256-278, 2006.

[Darwiche and Pearl, 1997] Adnan Darwiche and Judea Pearl. On the logic of iterated belief revision. Artificial Intelligence, 89:1-29, 1997.

[Fagin et al., 1995] Ronald Fagin, Joseph Halpern, Yoram Moses, and Moshe Vardi. Reasoning about knowledge. MIT Press, 1995.

[Fine, 1975] Kit Fine. Normal forms in modal logic. Notre Dame J. Formal Logic, 16:229-237, 1975.

[Hales et al., 2012] J. Hales, T. French, and R. Davies. Refinement quantified logics of knowledge and belief for multiple agents. In Proc. Advances in Modal Logic, 2012.

[Janin and Walukiewicz, 1995] D. Janin and I. Walukiewicz. Automata for the modal mu-calculus and related results. In Proc. Mathematical Foundations of Computer Science (MFCS), 1995.

[Katsuno and Mendelzon, 1991] Hirofumi Katsuno and Alberto $\mathrm{O}$. Mendelzon. On the difference between updating a knowledge base and revising it. In Proc. KR-91, pages 387-394, 1991.

[Kominis and Geffner, 2015] Filippos Kominis and Hector Geffner. Beliefs in multiagent planning: From one agent to many. Proceedings of the Twenty-Fifth International Conference on Automated Planning and Scheduling, pages 147-155, 2015. 
[McDermott et al., 1998] D. McDermott, M. Ghallab, A. Howe, C. Knoblock, A. Ram, M. Veloso, D. Weld, and D. Wilkins. PDDL- the planning domain definition language. Technical Report CVC TR98003/DCS TR1165, Yale Center for Computational Vision and Control, New Haven, CT, 1998.

[Miller and Muise, 2016] Tim Miller and Christian J. Muise. Belief update for proper epistemic knowledge bases. In IJCAI 2016, pages 1209-1215, 2016.

[Moss, 2007] Lawrence S. Moss. Finite models constructed from canonical formulas. Journal of Philosophical Logic, 36(6):605-640, 2007.

[Muise et al., 2015] Christian J. Muise, Vaishak Belle, Paolo Felli, Sheila A. McIlraith, Tim Miller, Adrian R. Pearce, and Liz Sonenberg. Planning over multi-agent epistemic states: A classical planning approach. In Proceedings of the Twenty-Ninth AAAI Conference on Artificial Intelligence, pages 3327-3334, 2015.

[Satoh, 1988] Ken Satoh. Nonmonotonic reasoning by minimal belief revision. In Proc. the First International Conference on Fifth Generation Computer Systems, 1988.

[ten Cate et al., 2006] Balder ten Cate, Willem Conradie, Maarten Marx, and Yde Venema. Definitorially complete description logics. In Proceedings, Tenth International Conference on Principles of Knowledge Representation and Reasoning, pages 79-89, 2006.

[To et al., 2011] Son Thanh To, Tran Cao Son, and Enrico Pontelli. Contingent planning as and/or forward search with disjunctive representation. Proceedings of the Twenty-First International Conference on Automated Planning and Scheduling, pages 258-265, 2011.

[van Ditmarsch et al., 2007] H. van Ditmarsch, W. van der Hoek, and B. P. Kooi. Dynamic epistemic logic. Springer, 2007.

[van Ditmarsch, 2001] Hans van Ditmarsch. Knowledge games. Bulletin of Economic Research, pages 249-273, 2001.

[Wan et al., 2015] Hai Wan, Rui Yang, Liangda Fang, Yongmei Liu, and Huada Xu. A complete epistemic planner without the epistemic closed world assumption. In Proceedings of the Twenty-Fourth International Joint Conference on Artificial Intelligence, pages 3257-3263, 2015.

[Winslett, 1988] Marianne Winslett. Reasoning about action using a possible models approach. In Proc. 7th AAAI Conference, 1988.

[Yu et al., 2013] Quan Yu, Ximing Wen, and Yongmei Liu. Multi-agent epistemic explanatory diagnosis via reasoning about actions. In Proceedings of the 23rd International Joint Conference on Artificial Intelligence, pages 11831190, 2013. 